\title{
GPPS-BJ-2019-0181
}

\section{INVESTIGATION OF THE UNSTEADY FLOW FEATURES IN A TIP LEAKAGE FLOW MODEL USING LARGE-EDDY SIMULATION}

\author{
Yanfei Gao ${ }^{2}$, Yangwei Liu ${ }^{1,2^{\star}}$ \\ 1. State Key Laboratory of Aerodynamics, China \\ Aerodynamics Research and Development Center, \\ P.O.BOX 211 Mianyang Sichuan 621000, China \\ 2. National Key Laboratory of Science and Technology \\ on Aero-Engine Aero-Thermodynamics, School of \\ Energy and Power Engineering, Beihang University, \\ Beijing, 100191, China \\ *Corresponding author: liuyangwei@126.com
}

\begin{abstract}
A simple tip leakage flow (TLF) model which consists of a square duct with a longitudinal slit on the top of a side wall is proposed to reproduce the jet flow/main flow shear mechanism of the tip leakage vortex (TLV) rolling-up in turbomachinery. Large-eddy simulation (LES) is employed to investigate the turbulence characteristics of the flow model under low Reynolds number condition. The geometry and boundary conditions of the flow model are simplified from a compressor. The vortex structures and turbulence characteristics of the LES results are compared with the measurements of the rotor. It is found that the flow model could reproduce similar flow field and turbulence structures compared with the TLF in the real rotor, thus it can be used to investigate the turbulence in practical flows. The turbulence anisotropy is investigated using Lumley triangle method, which shows different features in the TLV center and edge. The unsteadiness of turbulence is studied through velocity pulsation energy spectra. Low-frequency behaviours are believed to be the main source of the difference between different components and positions of turbulence distribution.
\end{abstract}

\section{INTRODUCTION}

The rotor tip leakage flow (TLF) is a typical flow in the turbomachinery which has overall effects on its performance (Denton, 1993). The TLF can be reduced to a wall jet flow in the tip clearance, which is driven by the pressure difference between the pressure side and the suction side of the blade. The leakage jet and the passage flow shear across each other, creating a helical rolling-up, which forms the tip-leakage vortex (TLV) (Lakshminarayana, 1995). The TLF has significant influences on the performance of turbomachinery, such as efficiency (Inoue et al., 1986), noise generation (Hsiao, 2005) and stability (Xie, et al. 2017).
Experimental investigations could be used to investigate flows in engineering, providing data on the pressure distribution, velocities, vorticities, and Reynolds stresses on specific points. Recently, PIV and SPIV methods are used to investigate the TLV and got impressive results on water pumps (Miorini et al. 2012; Wu et al. 2012) and axial compressors (Du et al. 2013; Li et al. 2017). The TLVs in all the above cases shows general turbulence characteristics, however it can hardly be explained by existing turbulence modelling methods. Researchers were trying to work out the turbulence mechanism by analysing its generation and transport nature over the last years (Miorini et al. 2012; Wu et al. 2012; Du et al. 2013). But it is still difficult to reconstruct the full three-dimensional flow field using experimental data, which brings limits to turbulence research. Reynolds-averaged Navier-Stokes (RANS) simulation, has been applied in turbomachinery for some time and made a very positive impact (Furukawa, 1999; Wells, 2010). However, turbulence models used in RANS method is a source of uncertainty (Liu, 2010). The nonequilibrium turbulent transport process and the anisotropy of Reynolds stresses are challenges to turbulence modelling (Gao, 2016). The full turbulence behavior can hardly be modelled accurately by existing turbulence models even though better models are being developed (Denton, 2010). To avoid the uncertainty caused by inaccurate turbulence modelling, higher fidelity methods are necessary. Direct numerical simulation (DNS), large eddy simulation (LES), and hybrid RANS/LES have been demonstrated to yield much more accurate results (You et al., 2007; Decaix et al., 2015; Scillitoe et al., 2017; Liu et al., 2017; Yan et al., 2018). Higher fidelity methods could be used to study turbulence physics and to educate RANS turbulence modelling (Liu et al., 2011; Lee et al., 2018; Kim et al., 2018; Tang et al., 2018). LES is a favorite and powerful tool for turbulence 
research because of much higher resolution compared with hybrid RANS/LES and much lower cost compared with DNS. But fine LES for turbomachinery is still very costly due to the complexity of the flow passages which requires considerable computational resources. Therefore, it is a feasible approach to investigate the fundamental turbulence physics in model problems rather than complicated industrial applications. The model problems abstracted from engineering flows are simpler but can reflect the main flow and turbulence characteristics.

In this paper, a physical flow model to simulate the TLF is proposed, which could reproduce the jet flow/main flow shear mechanism of the TLV roll-up. LES method is employed to predict the flow field of the flow model. The geometry and boundary conditions of the flow model are extracted from a real rotor. The performance of the flow model is verified by comparing the calculation results with measurements. Then the turbulence mechanism is analyzed using the LES results, including the turbulence anisotropy and spectra of turbulent velocity pulsations. Such investigations will not only be helpful to deepen the understanding of the turbulence mechanism, but also develop new insight for turbulence modelling in the TLF.

\section{METHODOLOGY}

The geometry of the blade passage is reduced to a square duct with a longitudinal slit on the top of a side wall. As shown in Figure 1, there is a main flow in the duct, resembling the axial flow in the turbomachinery, and a jet flow from the slit, resembling the tip leakage flow. Compared with the real rotor, the flow model mainly considers the jet-main flow interaction, but neglects other complex features of the TLF, such as the flows in the gap, the curvature of blade passage, the adverse pressure gradient, etc. The sizes in Figure 1 are summarized in Table 1.The side walls and top wall are set to no-slip walls, while the bottom is a symmetry plane. The main inlet and jet flow inlet are set to pressure inlet with total pressures of $1350 \mathrm{~Pa}$ and $1000 \mathrm{~Pa}$, respectively. The outlet on the right side is set to pressure outlet with a static pressure of 0 . The velocity directions of the inlet flows are normal to their respective boundaries. With the above settings, the mean velocities of the main inlet and jet are $42 \mathrm{~m} / \mathrm{s}$ and $37 \mathrm{~m} / \mathrm{s}$, respectively. Therefore, the Reynolds number ( $\mathrm{Re}$ ) based on the inlet velocity and slit length is approximately $4.2 \times 10^{5}$. The inlet turbulence is generated using spectral synthesizer method, with a turbulent intensity of $3.5 \%$ and the length scales are based on $L_{y}$ and $\tau$, respectively.

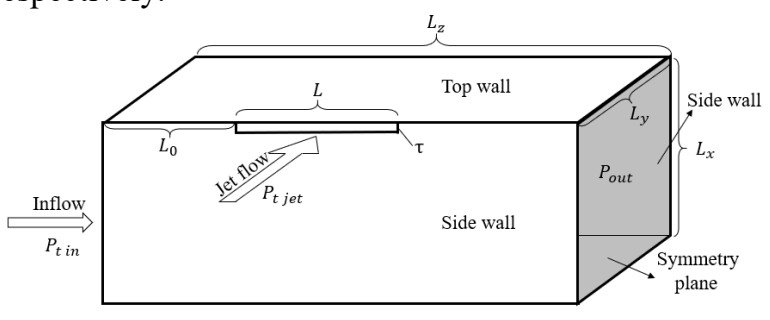

Figure 1 Geometry and boundary types of the physical model
Table 1 Sizes of the physical flow model

\begin{tabular}{|c|c|c|c|c|c|}
\hline$L_{x}$ & $L_{y}$ & $L_{z}$ & $L_{0}$ & $L$ & $\tau$ \\
\hline $100 \mathrm{~mm}$ & $100 \mathrm{~mm}$ & $400 \mathrm{~mm}$ & $100 \mathrm{~mm}$ & $150 \mathrm{~mm}$ & $2 \mathrm{~mm}$ \\
\hline
\end{tabular}

A single H-block mesh with $226 \times 275 \times 431(\mathrm{X} \times \mathrm{Y} \times$ $\mathrm{Z})$ cells is generated for LES, as shown in Figure 2. The mesh resolution on the wall is set to $y^{+}<1$ near the wall, and $x^{+}<30$ (pitchwise), $y^{+}<30$ (spanwise), $z^{+}<60$ (streamwise) away from the wall, which is fine enough for wall-resolved LES calculation. The mesh near the top wall especially in the jet shear layer is refined to get higher resolution in these regions.

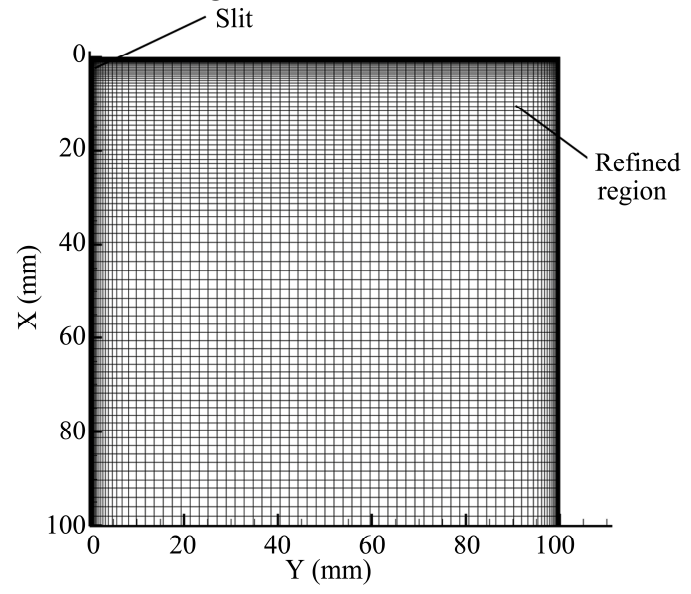

Figure 2 Grid distribution of the cross section in the flow model

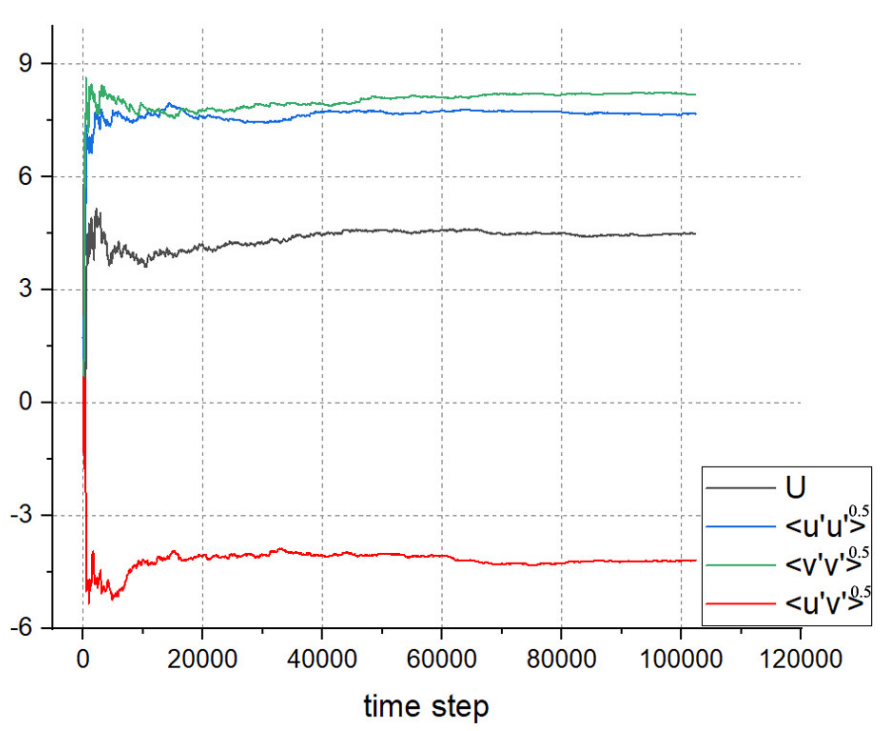

Figure 3 Statistical convergence result of first- and second-order momentum convergence history

The numerical simulations are carried out using the commercial software Ansys Fluent 16.1. The LES case employs dynamic Smagorinsky-Lilly model (Lilly, 1992) as the sub-grid scale model. Second-order bounded central differencing spatial discretization are applied for pressure and momentum respectively. The time step is set to $2 \times 10^{-6}$ second, to ensure the CFL number less than 0.5 . The results 
of the quantities are time-averaged over $1 \times 10^{5}$ timesteps after reaching statistically stationary state, which is $56 L / U_{\infty}$. Second-order upwind scheme is applied for the momentum and turbulence model equations. In the computation, cumulation method is used to obtain the statistical results, thus the convergence of the terms needs to be examined. Figure 3 shows the convergence history of first- and secondorder statistical quantities, the probe position is in the vortex area. It can be concluded that the first two order quantities have achieved statistical convergence.

The $I Q_{v}$ quality index can be used to evaluate the quality of LES mesh (Celik et al., 2009; Scillitoe et al., 2015):

$$
I Q_{v}=\left[1+\alpha_{v}\left(\left\langle\mu_{s g s}\right\rangle / \mu\right)^{n}\right]^{-1}
$$

where $\alpha_{v}=0.05$ and $n=0.53$. These constants are chosen so that $I Q_{v}$ ranges from 0 to 1 , with 0.8 considered a good LES resolution and 0.95 considered DNS. Figure 4 shows the subgrid-scale viscosity ratio in the vortex area, which can be used in the $I Q_{v}$ calculation. In most areas the viscosity ratio $\left\langle\mu_{s g s}\right\rangle / \mu$ is smaller than 6 , generating an $I Q_{v}$ larger than 0.88 , thus the mesh quality should satisfy the LES resolution.

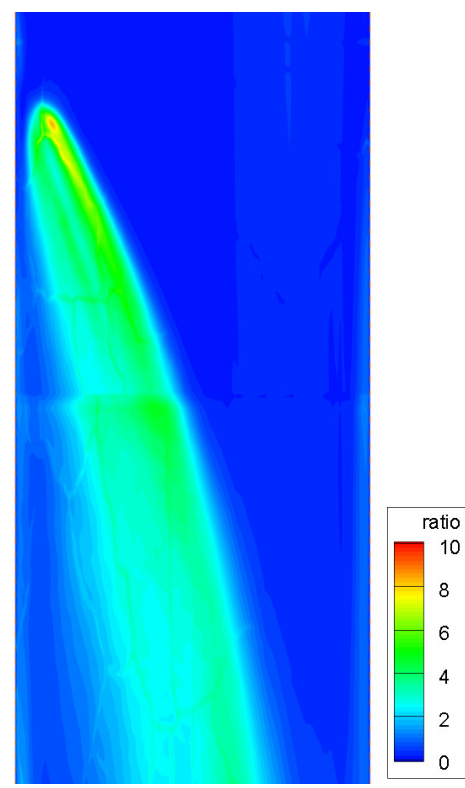

Figure 4 Subgrid-scale viscosity ratio, $x / T=4$

Then the anisotropy of the Reynold stress of these two methods are analyzed in the tip leakage flow using Lumley triangle method (Lumley, 1978), which derives the turbulence anisotropy quantities from the nondimensional form of the anisotropy tensor, as shown in Figure 4.

The Reynolds stress anisotropy status can be expressed using two coordinates, $\xi$ and $\eta$, which can be calculated by the following equations:

$$
\begin{gathered}
b_{i j}=\frac{\left\langle u_{i}^{\prime} u_{j}^{\prime}\right\rangle}{\left\langle u_{k}^{\prime} u_{k}^{\prime}\right\rangle}-\frac{1}{3} \delta_{i j} \\
\eta=\sqrt{\frac{1}{6} b_{i j} b_{i j}}
\end{gathered}
$$

$$
\xi=\sqrt[3]{\frac{1}{6} b_{i j} b_{j k} b_{k i}}
$$

and the three edges of the triangle are

$$
\begin{gathered}
\xi= \pm \eta \\
\eta=\left(\frac{1}{27}+2 \xi^{3}\right)^{1 / 2}
\end{gathered}
$$

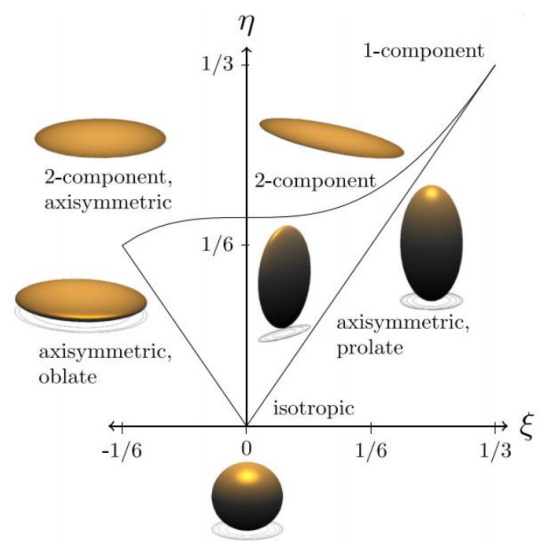

Figure 4 Schematic diagram of Lumley triangle and turbulence anisotropy

\section{RESULTS AND DISCUSSION}

The Q-criterion vortex identification result is shown in Figure 5. Strong vortexes in the jet region can be observed. The vorticity of the main TLV is negative, very strong at the beginning, and becomes weak while expanding downstream. Surrounding the main vortex there is a series of induced vortices with positive vorticity. Jet vortices exist in the jet flow between the wall and the main vortex structures, which will be involved into the TLV. Vortex breakdown phenomenon could be observed in the TLV. The vortex is distinct and stable upstream and loses stability downstream. The isolated main/induced vortex breakup into a series of small vortices.

A low-speed large-scale compressor stage was measured using SPIV method (Du et. al., 2013), which supplies abundant flow details in the rotor flow passage. In the following sections, the flow field in the physical flow model and real rotor will be compared to verify the performance of the flow model.

Figure 6 shows the time-averaged streamwise vorticity distributions in the flow model and the real rotor, both in $70 \%$ chord length sections. The lower edge of the jet flow has negative vorticity, while the upper edge, which is near the casing, has positive vorticity. Because the limitation of SPIV measurement, the region near the wall is not shown. The main TLV is an isolated vortex with negative vorticity, surrounded by positive vorticity area. Compared with Figure 5 , it could be concluded that the positive vorticity corresponding to the induced vortices. The relative locations and magnitudes of the vortexes in the chosen slices are 
comparable, indicating that the flow model could well reproduce the mean vortex structure in the rotor flow passage.

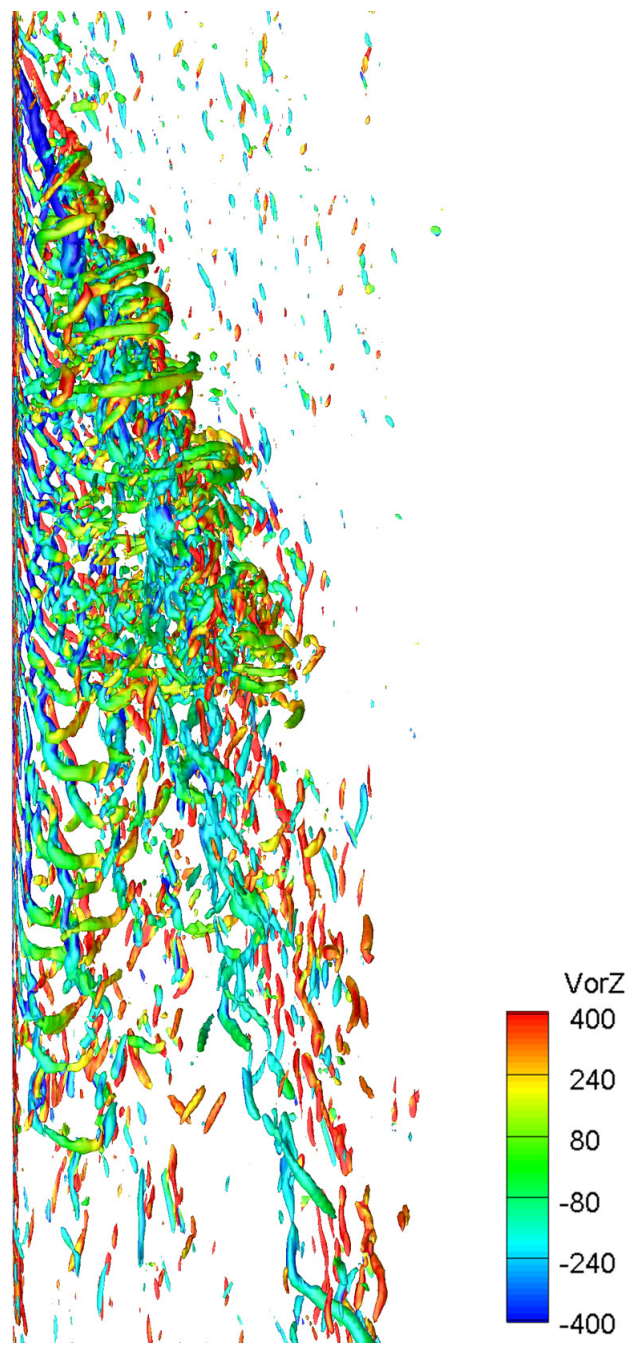

Figure 5 Iso-surface of $Q=2 E 7 \mathrm{~m}^{2} \mathrm{~s}^{-4}$, coloured by non-dimensionalized streamwise vorticity

The distributions of $\mathrm{k}$ in flow model simulation and real rotor measurement are shown in Figure 7. High-k area is mainly in the jet region and vortex region. A hot-spot could be observed in the centre of TLV in each result. This comparison shows that the overall turbulence structure in the two cases are similar. $\mathrm{k}$ consists of three components, the Reynolds normal stresses, $\left\langle u^{\prime} u^{\prime}\right\rangle,\left\langle v^{\prime} v^{\prime}\right\rangle$ and $\left\langle w^{\prime} w^{\prime}\right\rangle$, respectively, corresponding to the velocity pulsation in three directions. The distributions of $\mathrm{k}$ and Reynolds normal stresses in the flow model and real rotor along the line across the vortex core illustrated in Figure 7 are shown in Figure 8 and Figure 9. The vortex centres are located near $\mathrm{y}=0.032$, where the $\mathrm{k}$ is at its maximum. There is another smaller peak of $\mathrm{k}$ between $\mathrm{y}=0.04$ and 0.045 . The relative magnitudes of the three Reynolds normal stresses are slightly different in two cases. In the flow model, the $\left\langle u^{\prime} u^{\prime}\right\rangle$ is largest in the centre, followed by $\left\langle v^{\prime} v^{\prime}\right\rangle$ and $\left\langle w^{\prime} w^{\prime}\right\rangle$, while in the second peak, $\left\langle u^{\prime} u^{\prime}\right\rangle$ is the smallest, and $\left\langle v^{\prime} v^{\prime}\right\rangle$ has similar magnitude with $\left\langle w^{\prime} w^{\prime}\right\rangle$. In the real rotor measurement, the $\left\langle w^{\prime} w^{\prime}\right\rangle$ is small in the whole field. $\left\langle v^{\prime} v^{\prime}\right\rangle$ and $\left\langle u^{\prime} u^{\prime}\right\rangle$ have similar magnitude in the centre, and $\left\langle v^{\prime} v^{\prime}\right\rangle$ has dominant position in the second peak.
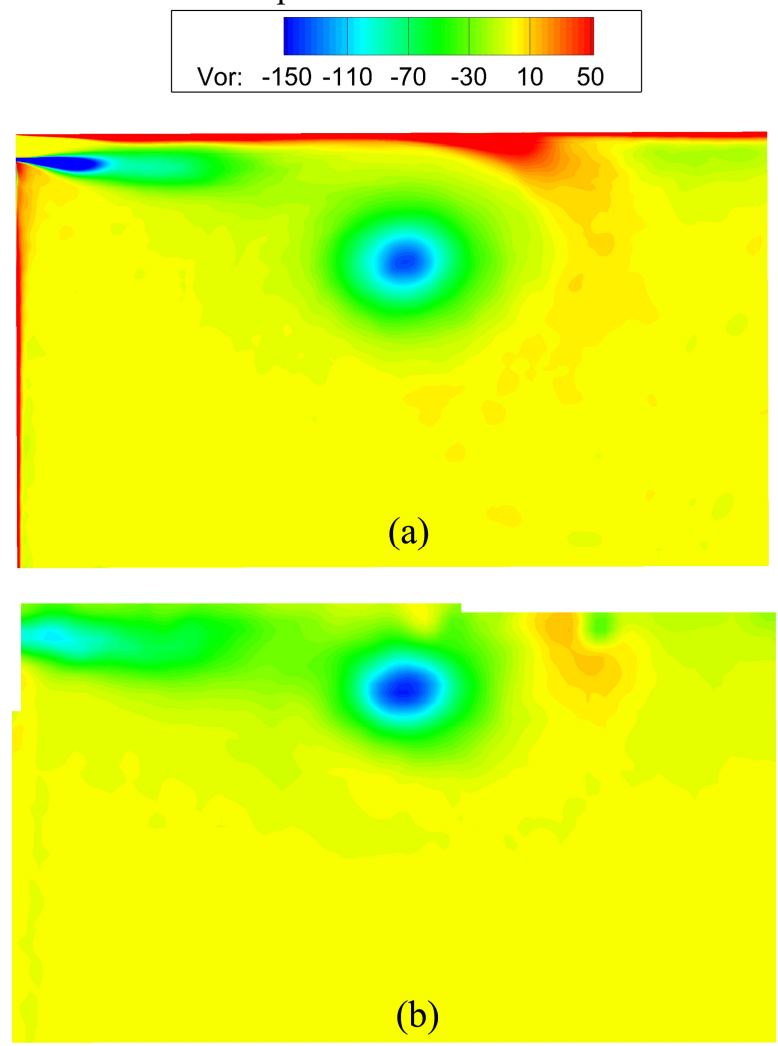

\section{Figure 6 Streamwise vorticity, non-dimensionalized by rotation rate, (a) flow model LES result, (b) real rotor measurement}
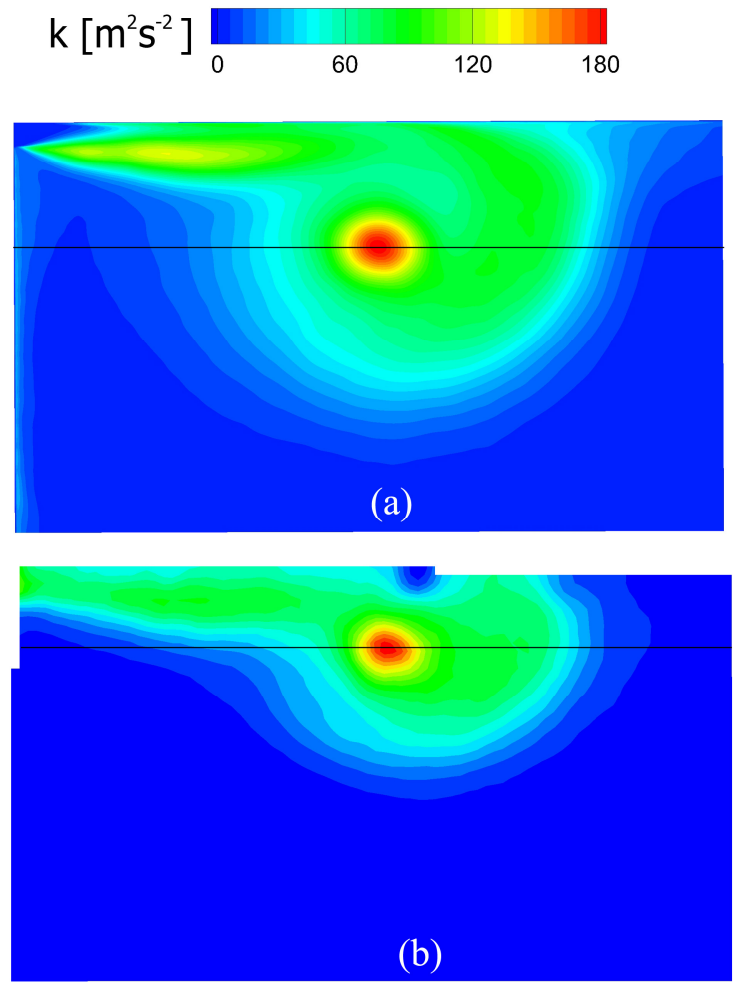
Figure 7 Distributions of $k$, (a) flow model LES result, (b) real rotor measurement

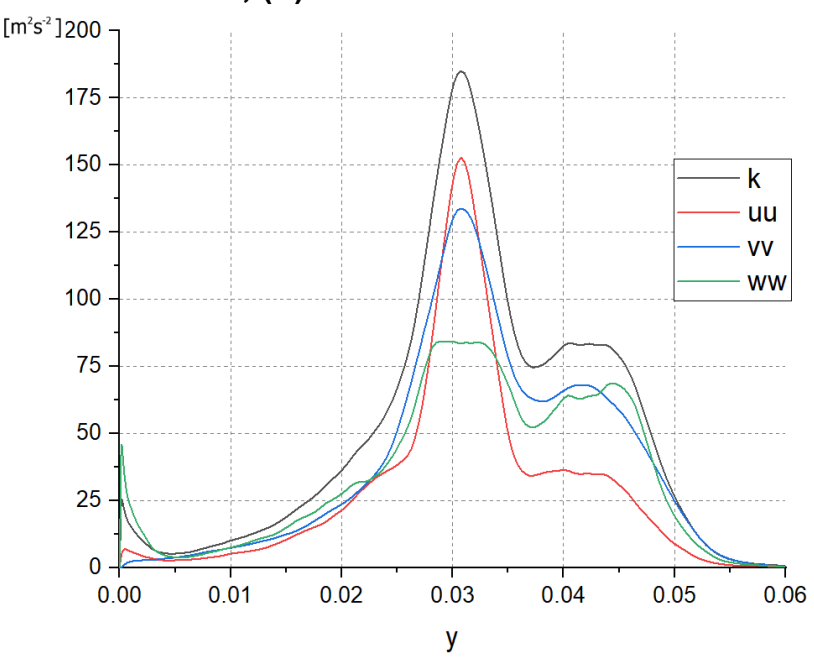

Figure $8 \mathrm{k}$ and Reynolds normal stresses in flow model LES result

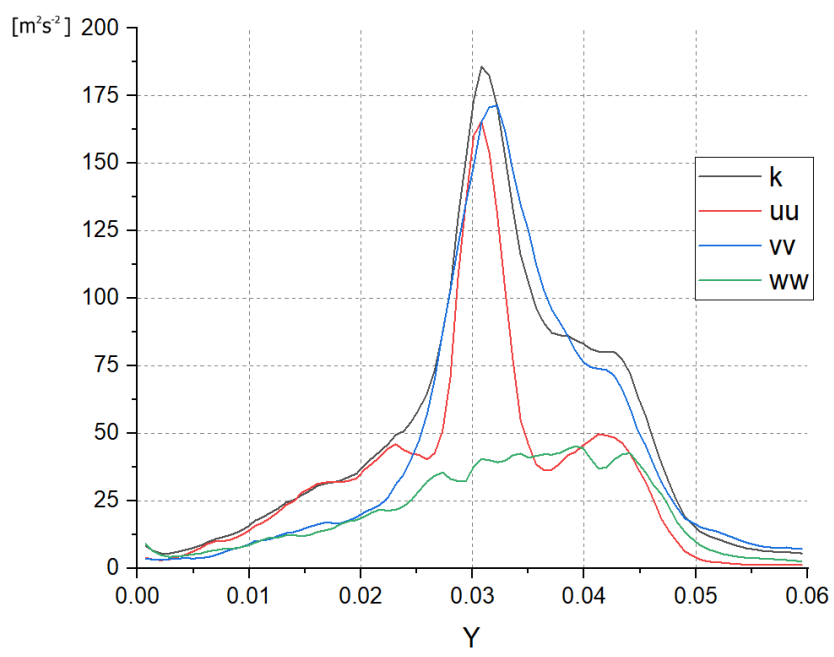

\section{Figure $9 \mathrm{k}$ and Reynolds normal stresses in real rotor measurement}

Apart from the Reynolds normal stress and $\mathrm{k}$, the Reynolds shear stresses could also prove the similarity of the turbulence structures between the flow model and the real rotor. u' and v' are more active in the TLV, thus the $\left\langle u^{\prime} v^{\prime}\right\rangle$ stresses in the two cases are shown in Figure 10. The $\left\langle u^{\prime} v^{\prime}\right\rangle$ is positive in the jet flow, shear layer surrounding the vortex and also in the centre of the TLV. Negative $\left\langle u^{\prime} v^{\prime}\right\rangle$ areas locate in both sides of the positive area, but with a smaller magnitude. The magnitudes and relative locations of the $\left\langle u^{\prime} v^{\prime}\right\rangle$ stresses in the two cases also show similarity, which further proves the performance of the flow model in simulating the real flow in rotor passage.

The anisotropy of Reynolds stress tensor is investigated using Lumley triangle parameters. Figure 11 shows the coordinates in the Lumley triangle reference, $\xi$ and $\eta$, respectively. $\xi$ reflects the shape of the anisotropy and $\eta$ reflects its level. The circles in the figures are the centres of the TLVs.
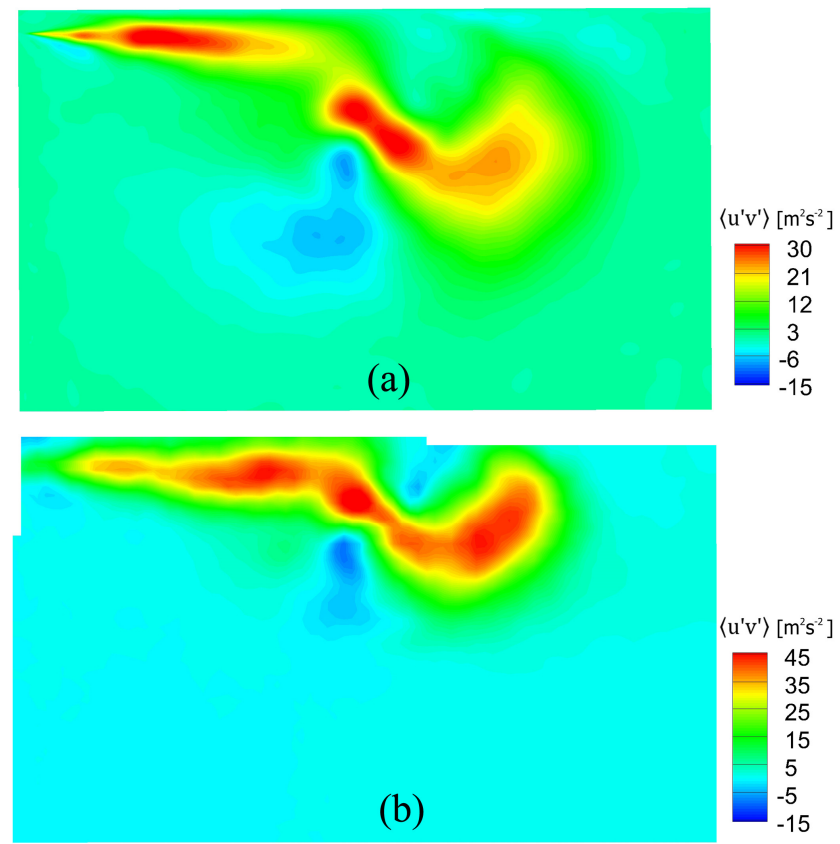

Figure 10 Distributions of Reynolds shear stresses $\left\langle u^{\prime} v^{\prime}\right\rangle$, (a) flow model LES, (b) real rotor measurement
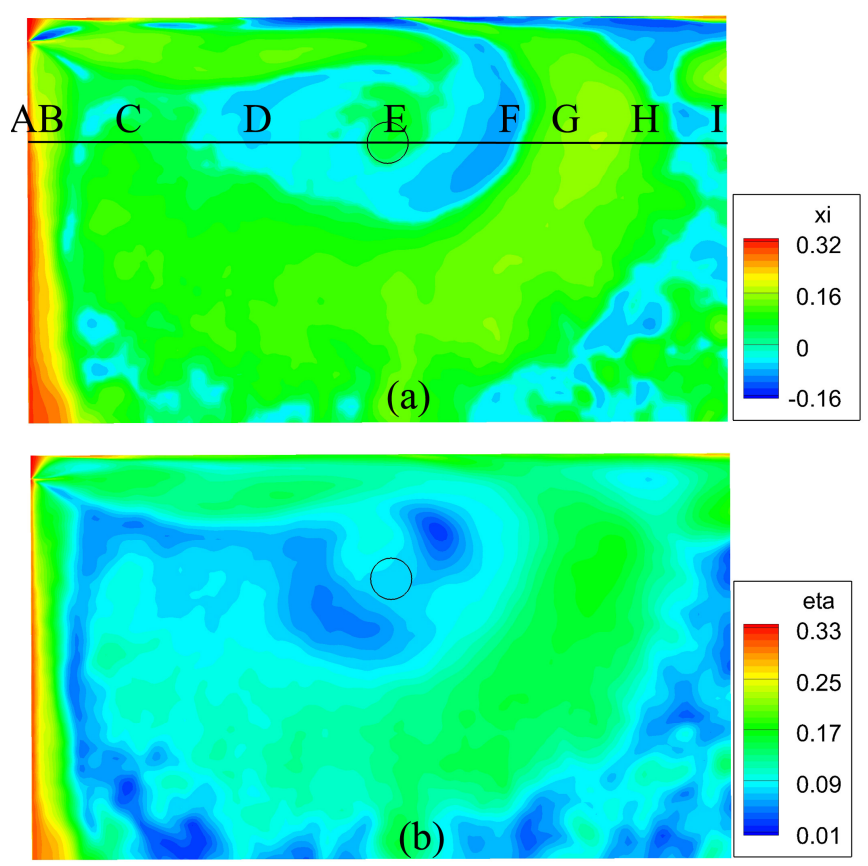

Figure 11 Distributions of Lumley triangle coordinates, (a) $\xi$, (b) $\eta$

In the side wall boundary layer, $\xi$ and $\eta$ are both large, indicating that the Reynolds stress is 1-dimensional. While in the TLV area, the anisotropy is relatively weak. The shape of the anisotropy tensor is more complicated. A line across the vortex centre (same line with Figure 8 and Figure 9) is extracted and the points along the line are plotted in Figure 12. 


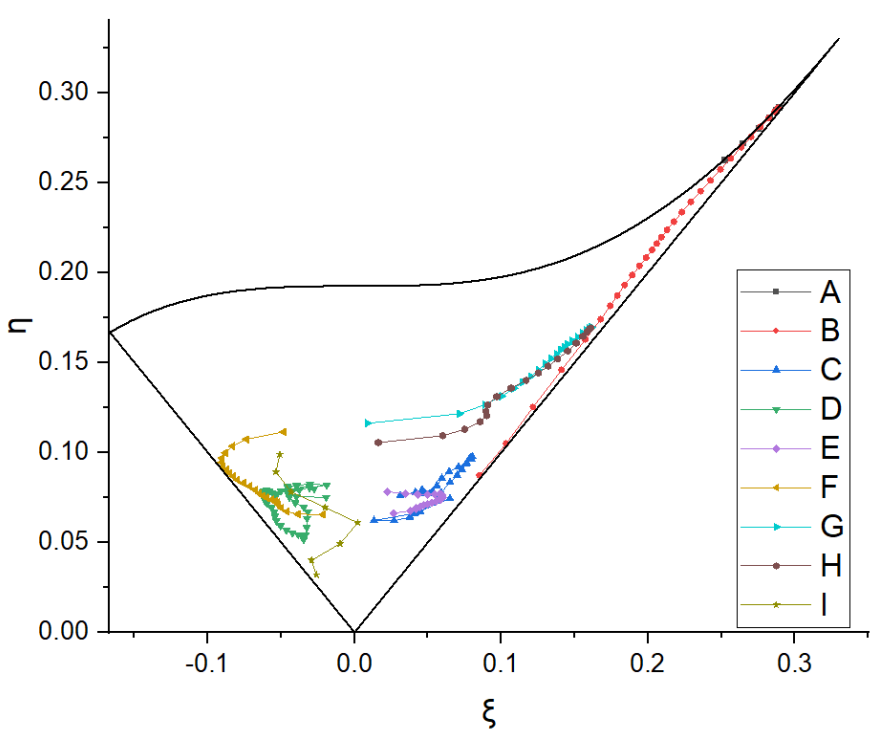

\section{Figure 12 Reynold stresses status in Lumley triangle along the line cross vortex centre}

Along the line shown in Figure 11(a), which is divided into 9 regions. Regions A and B are in the boundary layer thus the anisotropy is very strong in these regions. A is closer to the wall, where all velocity pulsations are weak, especially wall-normal pulsation is zero, thus the Reynolds stress shows a two-dimensional shape, with one dominant direction. In region $\mathrm{B}$, streamwise velocity as well as its pulsation becomes stronger, and the wall-normal pulsation becomes comparable to the pitchwise pulsation, thus the isotropy shows more single-dimensional. As the line goes into the flow, the $\eta$ becomes smaller and the points become more closer to the edge. In region $\mathrm{C}$, the points locate in the central area of the Lumley triangle, and $\xi$ is still positive, which means there still be a Reynolds stress superior to the others. In region $\mathrm{D}, \xi$ becomes negative while in region $\mathrm{E}$, it becomes positive again. The points in regions $\mathrm{C}$ and $\mathrm{E}$ are close to each other, as well as in regions D and F. From Figure 11, we can see that region $\mathrm{E}$ is in the vortex centre and regions $\mathrm{D}$ and $\mathrm{F}$ are symmetrical about the vortex centre. It can be concluded that the Reynolds stress tensor anisotropy is different inside the vortex region. Regions $G$ and $\mathrm{H}$ are near the edge of the vortex with high $\xi$ and $\eta$. Region I is near the main flow area, where there is little TLV influence. In conclusion, $\eta$ is high in boundary layer and outer region of the vortex, while $\xi$ shows positive-negative alternately.

Figure 13 and Figure 14 show the velocity pulsation energy spectra of two points, the vortex centre point and the vortex edge, corresponding to the two peaks in Figure 8. The slopes of the spectra have good agreement with $-5 / 3$ law in inertial subrange. In Figure 13, all three components are coincident in high-frequency range. While in low-frequency range, $\mathrm{w}$ pulsation is the weakest while $\mathrm{u}$ pulsation is slightly stronger that $v$. This indicates that the anisotropy of Reynolds stresses mainly come from the low-frequency range, while in inertial sub-region, turbulence shows isotropic. In Figure 14, the overall level of spectra is lower than that in Figure 13. u

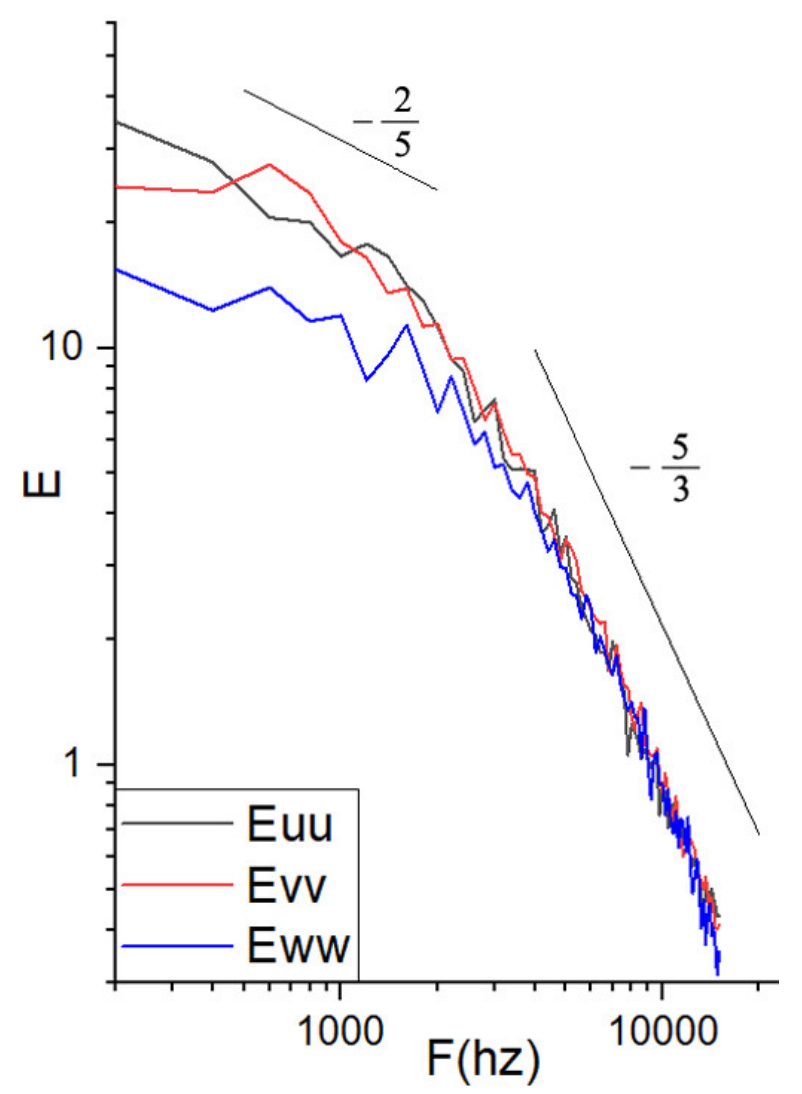

Figure 13 Energy spectra of the vortex centre point

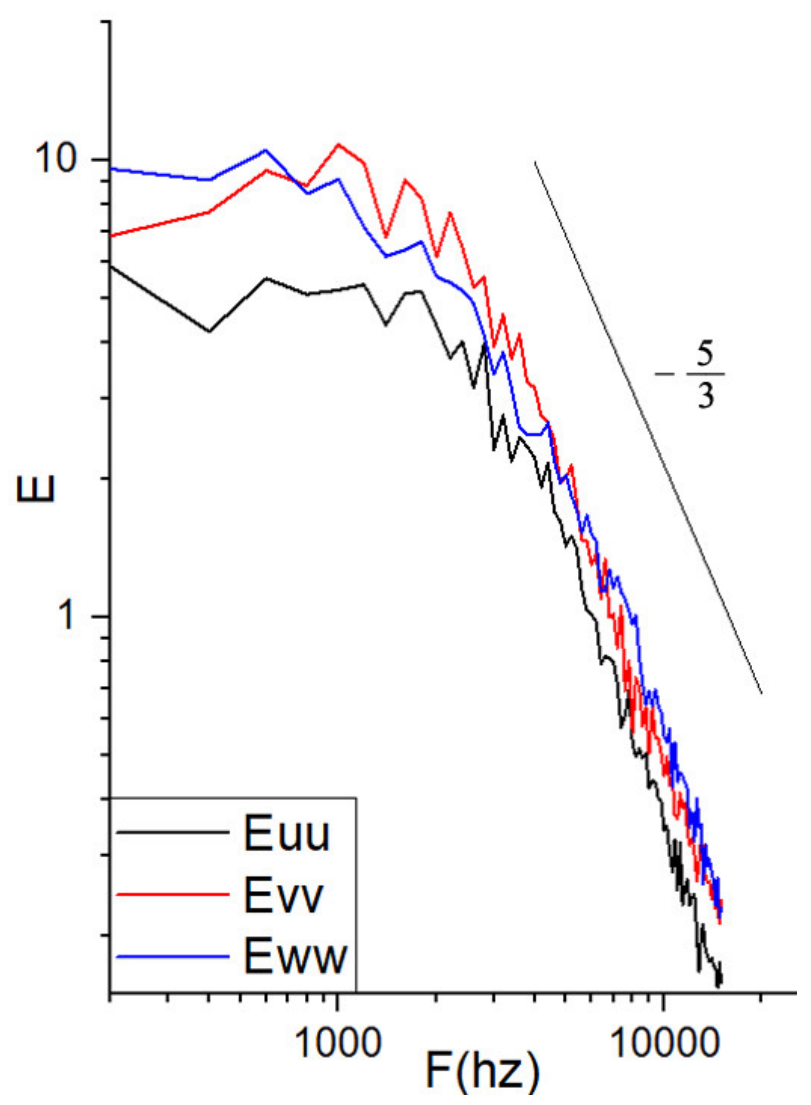

Figure 14 Energy spectra of the vortex edge point 
pulsation is the weakest, while $\mathrm{v}$ and $\mathrm{w}$ pulsations are superior in the middle and low frequency range, respectively. The distributions of energy spectra of different components and positions agree with the Reynolds normal stress distributions in Figure 8. Another difference between Figure 13 and Figure 14 is the slope of spectra in low-frequency range. In Figure 13, it has a slope of $-2 / 5$, while in Figure 14, it is almost flat. This indicates that the energy proportion of the energy region (low-frequency) is higher in the vortex centre than in the vortex edge.

\section{CONCLUSIONS}

A simplified physical flow model is proposed to simulate the tip leakage flow in turbomachinery. The flow model consists of a main flow in a square duct with a jet flow from a longitudinal slit, and the interaction of the flows will generate a streamwise vortex, resembling the tip leakage vortex (TLV). The flow model is investigated using LES method. The geometry and boundary conditions of the flow model are reduced from a real rotor with detailed experimental data, with simpler flow conditions at similar Reynolds number.

The LES results are compared with the measurements, which shows good agreement in mean velocity field and turbulent kinetic energy $(\mathrm{k})$. The streamwise vortex and induced vortex are predicted. The $\mathrm{k}$ has two peak regions in the cross section. One is in the center of the TLV, the other is in the curved shear layer of the leakage jet surrounding the vortex. The Reynolds normal stresses could also be compared to the measurements. The $\left\langle u^{\prime} u^{\prime}\right\rangle$ and $\left\langle v^{\prime} v^{\prime}\right\rangle$ stresses have similar magnitude, and both have a peak in the vortex center. The $\left\langle w^{\prime} w^{\prime}\right\rangle$ stress is relatively smaller than the other components. While the distributions of the shear stresses are very complicated, but the flow model could still grasp the main qualitative features. In general, the simplified flow model has the capacity to reproduce flow structure and turbulence characteristics resembling the real flow in the TLV.

Lumley triangle method is used to reveal the anisotropy features of Reynolds stress tensor. The two coordinates in Lumley triangle reference, $\eta$ and $\xi$, refer to the level and shape of the anisotropy. $\eta$ is high in boundary layer and outer region of the vortex, while $\xi$ shows positive-negative alternately. The Reynolds stress tensor anisotropy is different inside the vortex region.

The energy spectra of velocity pulsation of the vortex center point and edge point are investigated to show the frequency related characteristics of the turbulence in the TLV. The slopes of the spectra have good agreement with $5 / 3$ law in inertial subrange. The overall level of the energy spectra of different components and positions are corresponding to the Reynolds normal stress distributions. The anisotropy of Reynolds stresses mainly come from the low-frequency range, while in inertial sub-region, turbulence shows isotropic. Large scale vortices with lower-frequency pulsation contains more energy in the vortex centre compared with the edge point.

\section{NOMENCLATURE}

$\begin{array}{ll}L_{x}, L_{y}, L_{z} & \text { Dimensions of the flow model [mm] } \\ L & \text { Length of the slit [mm] } \\ \tau & \text { Width of the flow model [mm] } \\ L_{\infty} & \text { Reference length [mm] } \\ P_{t \text { in }} & \text { Inlet total pressure of the duct [Pa] } \\ P_{t j e t} & \text { Inlet total pressure of the jet slit [Pa] } \\ P_{\text {out }} & \text { Outlet static pressure of the duct [Pa] } \\ x^{+}, y^{+}, Z^{+} & \text {Non-dimensional turbulence wall distances } \\ X, Y, Z & \text { Cartesian coordinates } \\ U_{\infty} & \text { Reference velocity magnitude [m/s] } \\ U & \text { Time-averaged velocity } \\ u, v, w & \text { Filtered velocity components in LES } \\ \rho & \text { Density } \\ p & \text { Filtered pressure in LES } \\ r & \text { Radius of the rotor tip [m] } \\ \Omega & \text { Non-dimensional Rotation rate } \\ S & \text { Non-dimensional Strain rate } \\ Q & \text { Second invariant of velocity gradient } \\ \Omega_{z} & \text { Non-dimensional streamwise vorticity } \\ k & \text { Non-dimensional turbulent kinetic energy } \\ \varepsilon & \text { Turbulence dissipation rate } \\ \omega & \text { Specific dissipation rate } \\ \mu & \text { Molecular viscosity } \\ \mu_{t} & \text { Turbulence viscosity in RANS } \\ \mu_{s g s} & \text { Sub-grid scale viscosity in LES } \\ R e & \text { Reynolds number } \\ \varphi^{\prime} & \text { Fluctuating part of } \varphi \\ \langle\varphi\rangle & \text { Ensemble average of } \varphi \\ & \end{array}$

\section{ACKNOWLEDGMENTS}

We express our appreciation to the late Prof. Lipeng Lu, whose contribution to this work was of great significance. This work is supported by the National Natural Science Foundation of China (No. 51676007, No. 51420105008, No. 51790513), and also supported by the Open fund from State Key Laboratory of Aerodynamics. The authors would like to thank Prof. Baojie Liu and Dr. Xianjun Yu for providing experimental results.

\section{REFERENCES}

Celik I, Klein M, Janicka J. (2009). Assessment measures for engineering LES applications[J]. Journal of fluids engineering, 131(3): 031102.

Decaix J, Balarac G, Dreyer M, et al. (2015). RANS and LES computations of the tip-leakage vortex for different gap widths[J]. Journal of Turbulence, 16(4): 309-341.

Denton JD. (1993). Loss Mechanisms in Turbomachines [J]. Journal of Turbomachinery; 115(4):621-56.

Denton J D. (2010). Some limitations of turbomachinery CFD $[C] / / A S M E$ Turbo Expo 2010: Power for Land, Sea, and Air. American Society of Mechanical Engineers: 735-745.

Du H, Yu X, Zhang Z, et al. (2013). Relationship between the flow blockage of tip leakage vortex and its evolutionary procedures inside the rotor passage of a subsonic axial compressor[J]. Journal of Thermal Science, 22(6): 522-531. 
Furukawa M, Inoue M, Saiki K, et al. (1999). The Role of Tip Leakage Vortex Breakdown in Compressor Rotor Aerodynamics[J]. Journal of Turbomachinery, 121(3): 469480 .

Wells J, Salem Said A H, Ragab S. (2010). Effects of turbulence modeling on RANS simulations of tip vortices[C]/48th AIAA Aerospace Sciences Meeting Including the New Horizons Forum and Aerospace Exposition: 1104.

Hsiao C T, Chahine G L. (2005). Scaling of tip vortex cavitation inception noise with a bubble dynamics model accounting for nuclei size distribution[J].Journal of Fluids Engineering, 127(1): 55-65.

Inoue M, Kuroumaru M, Fukuhara M. (1986). Behavior of tip leakage flow behind an axial compressor rotor[J]. Journal of Engineering for Gas Turbines and Power, 108(1): 7-14.

Gao Y, Liu Y, Zhong L, et al. (2016). Study of the Standard k- $\varepsilon$ Model for Tip Leakage Flow in an Axial Compressor Rotor[J]. International Journal of Turbo \& JetEngines, 33(4): 353-360.

Kim S, Pullan G, Hall C A, et al. (2018). Stall inception in low pressure ratio fans[C]//ASME Turbo Expo 2018: Turbomachinery Technical Conference and Exposition. American Society of Mechanical Engineers: GT2018-75153.

Lakshminarayana B. (1995). Fluid dynamics and heat transfer of turbomachinery[M]. John Wiley \& Sons

Lee K B, Wilson M, Vahdati M. (2018). Validation of a Numerical Model for Predicting Stalled Flows in a LowSpeed Fan-Part I: Modification of Spalart-Allmaras Turbulence Model[J]. Journal of Turbomachinery, 140(5):051008.

Li, Yuanchao, Huang Chen, and Joseph Katz. (2017). "Measurements and Characterization of Turbulence in the Tip Region of an Axial Compressor Rotor." Journal of Turbomachinery 139.12: 121003.

Lilly D K. (1992). A proposed modification of the Germano subgrid-scale closure method[J]. Physics of Fluids A: Fluid Dynamics, 4(3): 633-635.

Liu Y, Yu X, Liu B. (2008). Turbulence models assessment for large-scale tip vortices in an axial compressor rotor[J]. Journal of Propulsion and Power, 24(1): 15-25.

Liu Y, Lu L, Fang L, et al. (2011). Modification of Spalart-Allmaras model with consideration of turbulence energy backscatter using velocity helicity[J]. Physics Letters A, 375(24): 2377-2381.

Liu, Y., Yan, H., Lu, L., and Li, Q. (2017). "Investigation of Vortical Structures and Turbulence Characteristics in Corner Separation in a Linear Compressor Cascade Using DDES," ASME J. Fluids Eng., 139(2), p. 021107.

Lumley, J. L. (1978). Computational Modeling of Turbulent Flows, Advances in Applied Mechanics, 18:123176.

Miorini, Rinaldo L., Huixuan $\mathrm{Wu}$, and Joseph Katz. (2012). "The internal structure of the tip leakage vortex within the rotor of an axial waterjet pump." Journal of Turbomachinery, 134.3: 031018.
Scillitoe A D, Tucker P G, Adami P. (2015). Evaluation of RANS and ZDES methods for the prediction of threedimensional separation in axial flow compressors[C]//ASME Turbo Expo 2015: Turbine Technical Conference and Exposition. American Society of Mechanical Engineers: V02BT39A041-V02BT39A041

Scillitoe A D, Tucker P G, Adami P. (2017). Numerical investigation of three-dimensional separation in an axial flow compressor: the influence of freestream turbulence intensity and endwall boundary layer state[J]. Journal of Turbomachinery, 139(2): 021011.

Tang Y, Liu Y, Lu L. (2018). Solidity effect on corner separation and its control in a high-speed low aspect ratio compressor cascade[J]. International Journal of Mechanical Sciences, 142: 304-321.

Wu, Huixuan, et al. (2012). "Turbulence within the tipleakage vortex of an axial waterjet pump." Aiaa Journal 50.11: 2574-2587.

Yan, H., Liu, Y., Li, Q., and Lu, L. (2018). “Turbulence Characteristics in Corner Separation in a Highly Loaded Linear Compressor Cascade," Aerosp. Sci. Technol., 75, pp. 139-154.

You D, Wang M, Moin P, et al. (2007). Large-eddy simulation analysis of mechanisms for viscous losses in a turbomachinery tip-clearance flow[J]. Journal of Fluid Mechanics, 586: 177-204.

Xie Z, Liu Y, Liu X, et al. (2017). Computational Model for Stall Inception and Nonlinear Evolution in Axial Flow Compressors[J]. Journal of Propulsion and Power, 34(3): 720-729. 